\title{
GHOST: GRADIENT HISTOGRAM OF SPECTRAL TEXTURE
}

\author{
Rui Jian Chu $^{a}$, Noel Richard ${ }^{a}$, Hermine Chatoux ${ }^{b}$, Christine Fernandez-Maloigne ${ }^{a}$, Jon Yngve Hardeberg ${ }^{c}$ \\ ${ }^{a}$ University of Poitiers, France \\ ${ }^{b}$ University of Burgundy, France \\ ${ }^{c}$ Norwegian University of Science and Technology, Norway
}

\begin{abstract}
A gradient-based texture feature for hyperspectral image is formulated with straightforward application to grayscale and color images. Processed in full band, GHOST is expressed as a four-dimensional probability density distribution encompassing joint metrological assessment of spectral and spatial properties. Its performance is close to Opponent Band Local Binary Pattern (OBLBP) in HyTexiLa texture classification (91\% - $99 \%$ accuracy) with feature size $0.2 \%$ of OBLBP's.
\end{abstract}

Index Terms - texture, metrology, spectral, gradient

\section{INTRODUCTION}

There is a plethora of texture feature extraction methods in the literature [1]. Few, however, defines what texture is. This results in numerous ad-hoc approaches which correlate little with metrology, despite attaining supreme accuracy in some specific tasks e.g. segmentation, classification and image retrieval. Consequently, one often finds difficulty in applying them for the metrology of industrial quality control or realtime remote sensing due to the lack of training dataset, complication in pre-processing and intricacy of parameter tuning.

In contrast, a metrological approach seeks to first define the measurand before performing the measurement. Therefore, the result is interpretable while qualities such as accuracy, bias and uncertainty are quantifiable. In the context of texture analysis, this means that such scheme is not constrained by the sensor properties; the texture difference should be invariant of its nature, be it grayscale, color, spectral or even beyond visible spectrum e.g. X-ray and fluorescence.

We note that images are inherently continuous despite the post-processed digital representation. As such, we choose to develop our assessment in hyperspectral context with straightforward application in grayscale and color domains. We first provide a texture definition in Sec. 2. Then, we devise a feature formulation as well as a measure for quantifying texture dissimilarity in Sec. 3. For validation, we present a texture classification scheme and result analysis in Sec. 4. Finally, in Sec. 5 we provide our concluding remarks for this work.

This work is supported by the French national projects ANR DigiPi and ERDF NUMERIC / e-Patrimoine.

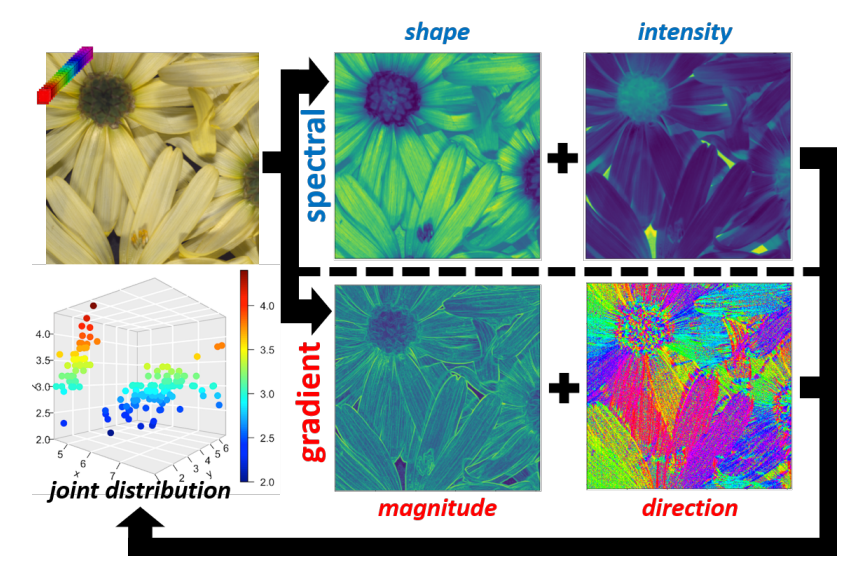

Fig. 1: An illustration of the concept of texture as a joint spectral-spatial distribution using spectral (shape, intensity) and gradient (texture "magnitude", direction) measures.

\section{TEXTURE DEFINITION}

Julesz pioneered the study of texture analysis in 1962 . He conjectured to discriminate texture based on $N^{\text {th }}$-order statistics, which is the probability to find at any spatial location a particular $N$-tuple for a given spatial relationship [2]. For example, the first-order statistics refers to the probability to find a particular pixel value, whereas the second-order statistics represents the probability to find a particular pixel pair for a given spatial relationship. Such consideration is applicable for any images be it of monovariate e.g. grayscale and X-ray or multivariate e.g. color, multispectral, and hyperspectral.

There has been numerous researches but no consensus on the optimum $N$ for preattentive visual discrimination $[3,4,5]$. However, they all imply the need for the joint consideration of spectral and spatial properties in texture recognition. This can be easily demonstrated from the fact that when the tonal variation is small, the dominant properties are the spectral ones; when the variation is large, the noticeable aspects are the spatial ones. In any cases, both kind of properties are always present and inextricable. Accordingly, we define texture as the joint distribution of spectral and spatial properties, with the later assessed via gradient measures in this work. 


\section{FEATURE FORMULATION}

\subsection{Assessing spatial properties}

Consider an image, $I: \mathbb{R}^{2} \rightarrow \mathbb{R}^{L}$ with $L$ bands, the gradient, $\nabla I(x)$ at a given position $x=\left(x_{1}, x_{2}\right)$ is given by:

$$
\nabla I(x)=\left[\begin{array}{llll}
\frac{\partial I_{1}(x)}{\partial x_{1}} & \frac{\partial I_{2}(x)}{\partial x_{1}} & \cdots & \frac{\partial I_{L}(x)}{\partial x_{1}} \\
\frac{\partial I_{1}(x)}{\partial x_{2}} & \frac{\partial I_{2}(x)}{\partial x_{2}} & \cdots & \frac{\partial I_{L}(x)}{\partial x_{2}}
\end{array}\right]^{T},
$$

which is really the Jacobian matrix. The spatial correlation matrix, $M$ is defined using full vector gradient (FVG) [6]:

$$
M(x)=\nabla I(x)^{T} G \nabla I(x)=\left[\begin{array}{ll}
M_{11}(x) & M_{12}(x) \\
M_{21}(x) & M_{22}(x)
\end{array}\right],
$$

where $G$ is the Gram matrix, necessary to take into account the non-orthogonality of spectral channels. It is defined using the scalar product for integrable functions:

$$
G=\left[\begin{array}{cccc}
\left\|S_{1}\right\|_{2}^{2} & \left\langle S_{1}, S_{2}\right\rangle_{2} & \ldots & \left\langle S_{1}, S_{L}\right\rangle_{2} \\
\left\langle S_{2}, S_{1}\right\rangle_{2} & \left\|S_{2}\right\|_{2}^{2} & \ldots & \left\langle S_{2}, S_{L}\right\rangle_{2} \\
\vdots & \vdots & \ddots & \vdots \\
\left\langle S_{L}, S_{1}\right\rangle_{2} & \ldots & \left\langle S_{L}, S_{L-1}\right\rangle_{2} & \left\|S_{L}\right\|_{2}^{2}
\end{array}\right],
$$

where $S_{i}$ is the spectral sensitivity function for the $i^{\text {th }}$ band.

The eigenvalues, $\lambda_{ \pm}$of $M$ and eigenvectors ${ }^{1}$ oriented at angle $\theta_{ \pm}$such that $\theta_{-}=\theta_{+}+(\pi / 2)$ is given by [7]:

$$
\begin{aligned}
& \lambda_{ \pm}=-\frac{1}{2}\left(\operatorname{tr}(M) \pm \sqrt{\operatorname{tr}(M)^{2}-4|M|}\right), \\
& \theta_{+}=\operatorname{sgn}\left(M_{12}\right) \arcsin \left(\frac{\lambda_{+}-M_{11}}{2 \lambda_{+}-\operatorname{tr}(M)}\right)^{\frac{1}{2}},
\end{aligned}
$$

where $\operatorname{sgn}(\cdot),|\cdot|$ and $\operatorname{tr}(\cdot)$ are the sign function, matrix determinant and trace respectively. To interpret $\lambda_{ \pm}$and $\theta_{ \pm}$(see Fig. 2), we first introduce the directional derivative, $\nabla_{\theta} I(x)$ :

$$
\nabla_{\theta} I(x)=\nabla I(x) \cdot \hat{u}_{\theta},
$$

where $\hat{u}_{\theta}$ is an unit vector oriented at angle $\theta$. The spatial correlation matrix, $M$ and $\nabla_{\theta} I(x)$ are related by:

$$
\left\|\nabla_{\theta} I(x)\right\|_{2}^{2}=\hat{u}_{\theta}^{T} M(x) \hat{u}_{\theta} .
$$

Thanks to the eigendecomposition of $M$, we note that the quadratic form $\left\|\nabla_{\theta} I(x)\right\|_{2}^{2}$ can also be expressed as:

$$
\left\|\nabla_{\theta} I(x)\right\|_{2}^{2}=\lambda_{+} \cos \left(\theta_{+}-\theta\right)+\lambda_{-} \sin \left(\theta_{+}-\theta\right),
$$

For $k \in \mathbb{Z}$, the extrema of $\left\|\nabla_{\theta} I(x)\right\|_{2}=\sqrt{\left\|\nabla_{\theta} I(x)\right\|_{2}^{2}}$ are:

$$
\begin{aligned}
& \max _{0 \leq \theta<2 \pi}\left\|\nabla_{\theta} I(x)\right\|_{2}=\left\|\nabla_{\left(\theta_{+}+k \pi\right)} I(x)\right\|_{2}=\sqrt{\lambda_{+}}, \\
& \min _{0 \leq \theta<2 \pi}\left\|\nabla_{\theta} I(x)\right\|_{2}=\left\|\nabla_{\left(\theta_{-}+k \pi\right)} I(x)\right\|_{2}=\sqrt{\lambda_{-}},
\end{aligned}
$$

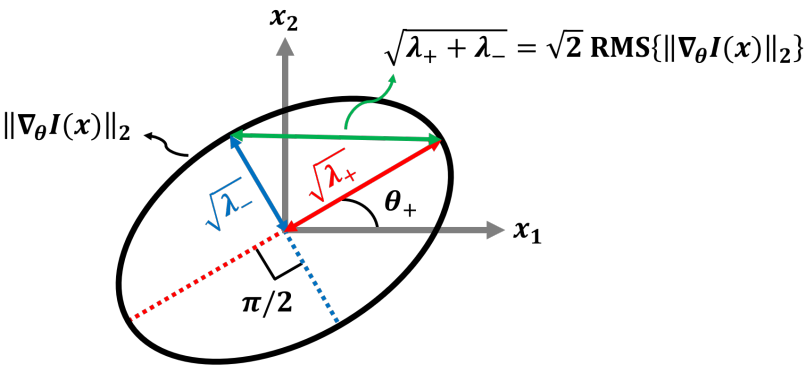

Fig. 2: Geometric interpretation of $\lambda_{ \pm}$and $\theta_{ \pm}$in the context $\left\|\nabla_{\theta} I(x)\right\|_{2}$, where $\nabla_{\theta} I(x)$ is the directional derivative.

with its root mean square, RMS $\left\{\left\|\nabla_{\theta} I(x)\right\|_{2}\right\}$ given by:

$$
\left(\frac{1}{2 \pi} \int_{0}^{2 \pi}\left(\left\|\nabla_{\theta} I(x)\right\|_{2}\right)^{2} d \theta\right)^{\frac{1}{2}}=\sqrt{\lambda_{+}+\lambda_{-}} / \sqrt{2} .
$$

Three kinds of local structure can be inferred from $\lambda_{ \pm}$: $\lambda_{+} \gg \lambda_{-}$indicates an edge; $\lambda_{ \pm} \gg 0$ indicates a corner; $\lambda_{+} \approx \lambda_{-} \approx 0$ indicates uniformity [8]. Correspondingly, one may be prompted to measure texture in terms of "edgelikeness" and thus use $\lambda_{+}$[9] or $\lambda_{+}-\lambda_{-}$[10]. Alternatively, one may choose to measure texture in terms of "cornerlikeness" using $\lambda_{-}$[11]. If both edge-likeness and cornerlikeness are considered, then one could choose $\lambda_{+}+\lambda_{-}$[6].

We propose to measure the spatial properties in terms of texture "magnitude" (degree of texturedness) and direction. To measure texture "magnitude" (hereby denoted as $T$ ), we consider the following possibilities ${ }^{2}: f\left(\lambda_{+}\right), f\left(\lambda_{-}\right), f\left(\lambda_{+}+\right.$ $\left.\lambda_{-}\right)$and $f\left(\lambda_{+}-\lambda_{-}\right)$. We analyze them if they fulfill all of the following criteria which are expected from a valid measure:

- In the absence of texture i.e. $\left\|\nabla_{\theta} I(x)\right\|_{2}=0, T=0$ (discounting the effect of noise): violated by none.

- In the presence of texture, $T \neq 0$ : violated by $f\left(\lambda_{-}\right)$ and $f\left(\lambda_{+}-\lambda_{-}\right)$as they can be null for texture made up of only edges and corners respectively.

- At a given scale, $T$ should have higher values for images more textured than that of less textured: violated by $f\left(\lambda_{+}\right), f\left(\lambda_{-}\right)$and $f\left(\lambda_{+}-\lambda_{-}\right)$as illustrated in Fig. 3. The ignorance of $\lambda_{-}$by $f\left(\lambda_{+}\right)$induces a lack in assessment as the pixel variation in directions other than the maximum's $\left(\theta_{+}\right)$are not taken into account. Likewise, $f\left(\lambda_{-}\right)$does not consider directions other than the minimum's $\left(\theta_{-}\right)$, while $f\left(\lambda_{+}-\lambda_{-}\right)$cancels out when $\lambda_{+}=\lambda_{-}$e.g. for corners of equal strength.

\footnotetext{
${ }^{1} \theta_{ \pm}$are undefined if $\operatorname{tr}(M)^{2}-4|M|=0$ because then $\left\|\nabla_{\theta} I(x)\right\|_{2}^{2}$ (related to change of pixel values) is the same in any direction as $\lambda_{+}=\lambda_{-}$. Fortunately, studies show that such case is unlikely in practical natural images due to noise, quantization level and sensor characteristics [7].

${ }^{2}$ The notation $f(y)$ means "the function of" e.g. $2 y, y / 2, \sqrt{y}$ etc.
} 


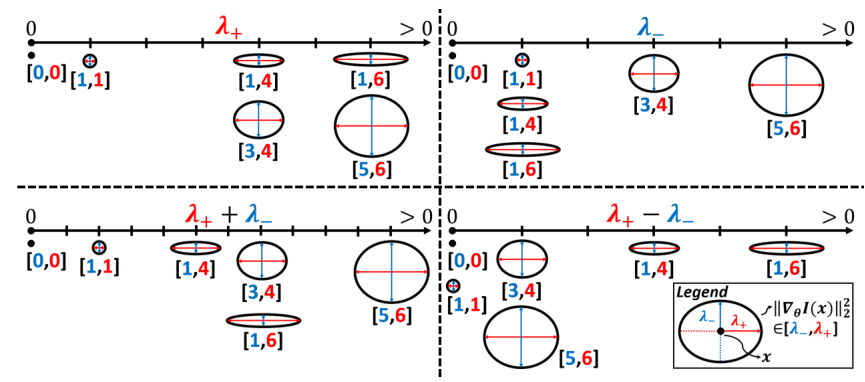

Fig. 3: Specified by $\left\|\nabla_{\theta} I(x)\right\|_{2}^{2}$ (visualized as black ellipse), the pixels are ranked according to their texture "magnitude" based on (a) $\lambda_{+}$, (b) $\lambda_{-}$, (c) $\lambda_{+}+\lambda_{-}$and (d) $\lambda_{+}-\lambda_{-}$.

Clearly, $f\left(\lambda_{+}+\lambda_{-}\right)$is the only one that fulfill all the criteria set. Also supported by the fact that it is dissociated from directionality by definition (see Eq. 9), we select $T=$ $\sqrt{\lambda_{+}+\lambda_{-}} / \sqrt{2}$ as the measure for texture "magnitude". As for texture direction, the obvious choice is $\theta_{+}$as it refers to the direction on which the pixel variation is the maximum. If the texture is isotropic, then the distribution of $\theta_{+}$is uniform, indicating non-directionality. We therefore express our measure of the spatial properties, hereby denoted as $\mathcal{J}_{N}$, as the probability to find at any spatial location a particular texture "magnitude" and direction. Computing the gradient using a $N=N^{\prime} \times N^{\prime}$ filter, we express $\mathcal{J}_{N}$ as the probability density function (PDF) of the continuous random variables $T$ and $\theta_{+}$:

$$
\mathcal{J}_{N}\left(T, \theta_{+}\right)=P\left(\nabla I(x)=\left(T, \theta_{+}\right)\right),
$$

with the subscript of " $N$ " due to its relationship with $N^{\text {th }}$ order statistics as defined in Julesz conjecture [2].

\subsection{Assessing spectral properties}

To quantify the spectral distribution is to construct a spectral histogram of $P(s)$, akin to the concept of image histogram where $s$ is a given spectrum. However, due to the high dimensionality of hyperspectral data, it is computationally infeasible to directly define $P(s)$. Consider a marginal quantization of $Q$ levels for each of the $L$ channels, a histogram of $Q^{L}$ bins would be required which is memory extensive.

As solution, we transpose the formulation in difference space. In particular, we represent $I(x)=s \in \mathbb{R}^{L}$ by spectral difference, $\Delta\left(s, s^{\prime}\right) \in \mathbb{R}^{L^{\prime}}$ to a spectral reference, $\hat{s}$ such that $L^{\prime} \ll L$. Using Kullback-Leibler pseudo-divergence (KLPD) [12], the difference between two spectra, $s$ and $s^{\prime}$ is:

$$
\begin{aligned}
\Delta\left(s, s^{\prime}\right) & =\Delta G\left(s, s^{\prime}\right)+\Delta W\left(s, s^{\prime}\right), \\
\Delta G\left(s, s^{\prime}\right) & =\|s\|_{1} \cdot \operatorname{KL}\left(\bar{s} \| \bar{s}^{\prime}\right)+\left\|s^{\prime}\right\|_{1} \cdot \operatorname{KL}\left(\bar{s}^{\prime} \| \bar{s}\right), \\
\Delta W\left(s, s^{\prime}\right) & =\left(\|s\|_{1}-\left\|s^{\prime}\right\|_{1}\right) \log \left(\frac{\|s\|_{1}}{\left\|s^{\prime}\right\|_{1}}\right),
\end{aligned}
$$

where $\Delta G$ and $\Delta W$ are the intensity and shape differences respectively. While $\operatorname{KL}(\cdot \| \cdot)$ is the Kullback-Leibler diver- gence, $\bar{s}$ is the normalized spectrum with $\|s\|_{1}=\int s(\lambda) d \lambda$. We therefore express our measure of the spectral properties as the probability to find at any spatial location a particular spectral difference with respect to the selected spectral reference. Such measure, hereby denoted as $\mathcal{J}_{1}$ can then be expressed as a PDF of the continuous random variables $\Delta G$ and $\Delta W$ [13]:

$$
\mathcal{J}_{1}^{\hat{s}}(\Delta G, \Delta W)=P(\Delta(I(x), \hat{s})=(\Delta G, \Delta W)),
$$

with the subscript of "1" due to its relationship with first-order statistics as defined in Julesz conjecture [2].

\subsection{The texture feature and difference measure}

In accordance to our definition in Sec. 2, we propose a texture feature, $\mathcal{J}$ embedding a simultaneous measurement of the spectral and spatial properties. It expresses the probability to find at any spatial location a particular spectral difference to a reference and a particular texture "magnitude" as well as direction. As a four-dimensional PDF, $\mathcal{J}$ is expressed as:

$$
\begin{aligned}
& \mathcal{J}^{\hat{s}}\left(\Delta G, \Delta W, T, \theta_{+}\right) \\
& =P\left(\Delta(I(x), \hat{s})=(\Delta G, \Delta W) \wedge \nabla I(x)=\left(T, \theta_{+}\right)\right),
\end{aligned}
$$

which we name as Gradient Histogram Of Spectral Texture (GHOST). Despite its development in hyperspectral context, it should be clear that GHOST is applicable to grayscale and color images with adapted gradient and difference measures.

For texture dissimilarity measurement, we use Jeffrey's divergence, which is the symmetric version of KullbackLeibler divergence (KLD) [14]. Due to the multi-dimensionality of GHOST, it is computationally expensive to process KLD directly based on histogram modeling. As an alternative, we use Gaussian mixture model (GMM) to statistically model the feature. As there exists no close form solution for KLD between two GMMs, we use variational approximation [15].

\section{RESULT AND DISCUSSION}

\subsection{Texture classification and feature computation}

We assess GHOST's performance by applying texture classification on HyTexiLa [16]. Consisting of 112 images measuring $1024 \times 1024$ with $L=186$ spectral bands ranging from $405.37 \mathrm{~nm}$ to $995.83 \mathrm{~nm}$, HyTexiLa is a collection of texture from food (10 images), stone (4 images), textile (65 images), vegetation (15 images) and wood (18 images). We split each image into 25 patches with a train:test ratio of $12: 13$ and perform nearest neighbor classification. To avoid bias, we repeat both intra-categorical and inter-categorical classification 100 times and report the average accuracy with standard deviation.

In this work, we compute GHOST using a $3 \times 3$ filter constructed using a Gaussian function and its derivative as defined in [6]. We use the same reference as the one used in [13]. 


\begin{tabular}{|c|c|c|c|}
\hline Category & A. spectrum & GHOST & OBLBP \\
\hline Food & $96.3 \pm 0.5$ & $97.9 \pm 0.9$ & $99.9 \pm 0.3$ \\
Stone & $86.7 \pm 1.2$ & $99.2 \pm 1.2$ & $99.9 \pm 0.3$ \\
Textile & $98.7 \pm 0.1$ & $99.2 \pm 0.2$ & $99.9 \pm 0.1$ \\
Vegetation & $83.9 \pm 0.6$ & $94.6 \pm 2.0$ & $97.4 \pm 1.2$ \\
Wood & $78.9 \pm 0.7$ & $91.0 \pm 3.1$ & $94.2 \pm 2.5$ \\
All & $91.4 \pm 0.3$ & $96.1 \pm 0.9$ & $98.4 \pm 0.6$ \\
\hline
\end{tabular}

Table 1: Comparison of texture classification accuracy using average spectrum (baseline), GHOST, and OBLBP. "All” denotes inter-classification involving all 112 HyTexiLa images.

We compare our performance with that of spectral classification (baseline) using average spectrum as well as OBLBP [16]. The average spectrum is defined marginally for each of the patches. On the other hand, OBLBP is basically LBP considered in the context of cross-channel processing for hyperspectral application. The LBP is constructed considering $J=8$ pixels in a circular neighborhood of radius $r=1$.

Modeled using 8 GMM components (as optimally determined based on Bayesian Information Criterion), GHOST is processed in full-band whereas OBLBP is processed on a reduced set of spectral bands, $\widetilde{L}=18$. The feature size for GHOST and OBLBP are 168 and 82944 scalars respectively.

\subsection{Subjective evaluation: Milkcoffee and Wood}

We analyze the texture content of two hyperspectral images: Milkcoffee and Wood as shown in Fig. 4 (a) and (b) respectively. On spectral properties, we observed that Milkcoffee is more spectrally homogeneous in contrast to Wood which is more spectrally diverse with alternating strips of different color appearance. This is reflected in the larger spread of $\Delta G$ for Wood compared to that of Milkcoffee. On the other hand, Milkcoffee is visually darker while Wood is brighter. Depending on the choice of spectral reference, one will have larger $\Delta W$ than the other. In this case, clearly the reference is at similar intensity level with Wood, hence the lower values of $\Delta W$ for Wood and higher values for Milkcoffee. On spatial properties, Milkcoffee is less textured than Wood and isotropic, hence its lower $T$ values in average and the uniform distribution of $\theta_{+}$. In comparison, Wood is highly textured with a directional variation, hence its higher $T$ values with $\theta_{+}$peaked around 0. Evidently, GHOST is able to capture the complexity of spectral-spatial properties in an extremely efficient, compact and interpretable manner.

\subsection{Comparison with spectral classification and OBLBP}

OBLBP considers only the sign of pixel difference in a circular neighborhood. As a result, information about the magnitude and permutation is lost. In comparison, GHOST records gradient ( $T$ and $\theta_{+}$) in a continuous manner without any

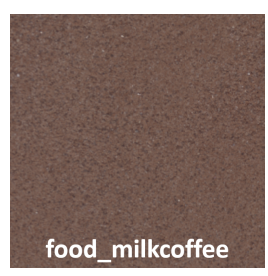

(a)

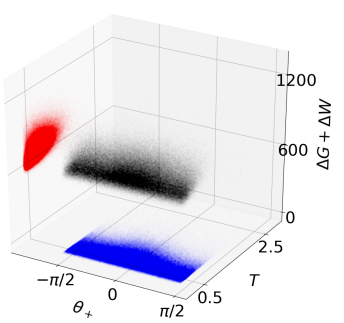

(c)

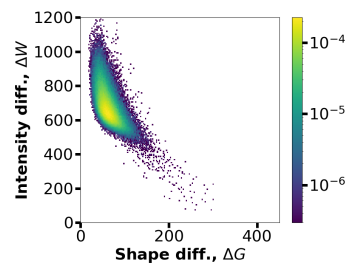

(e)

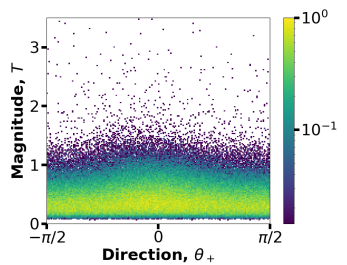

(g)

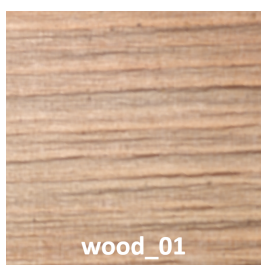

(b)

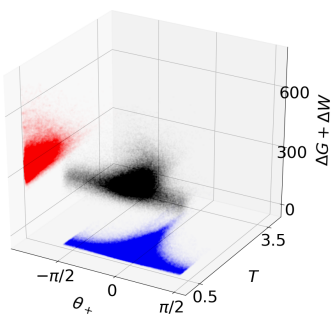

(d)

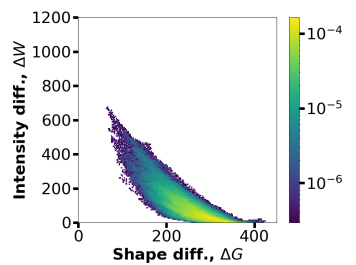

(f)

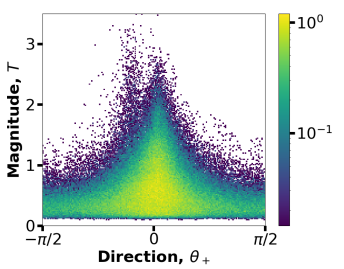

(h)
Fig. 4: Two hyperspectral textures, illustrated in color (row 1) with their GHOST in black (row 2, not plotted in the same scale). For a three-dimensional view, the dimensions of $\Delta G$ and $\Delta W$ are collapsed into one using their sum. The red and blue plots are projections on the $(T, \Delta G+\Delta W)$ and $\left(\theta_{+}, T\right)$ planes respectively. For detailed assessment, $(\Delta G, \Delta W)$ and $\left(\theta_{+}, T\right)$ are plotted in row 3 and 4 respectively.

quantization, preserving the integrity of the information extracted. Thanks to the probabilistic construct, a natural and metrological texture similarity measure is thus possible.

From Table 1, it can be seen that GHOST performs well in HyTexiLa classification. It is able to correctly classify at least $91 \%$ of the textures in each category. Its performance clearly overpasses that of spectral classification (using average spectrum) whereas OBLBP achieves the highest classification accuracy in overall. Among other factors, we attribute our lower performance mainly to the GMM modeling. Obviously, the efficiency of GHOST is subjected to adequate sta- 
tistical modeling by GMM. It is quite possible that GMM fails to capture the complexity of the distribution, thus causing its lower discriminability. On the other hand, for simplicity we use the same number of components for every texture. In reality, each texture might need different number of components for adequate modeling. Besides, the choice of filter for the gradient computation may yet be optimized. Moreover, the spectral reference, by which GHOST's performance is highly affected, is also selected empirically at this stage.

Nevertheless, the excellent performance of OBLBP is tainted by its huge feature size (500 times larger than GHOST). As OBLBP is formed by concatenation of $J$-bits histogram at each channel (total bin $=2^{J} \cdot \widetilde{L}^{2}$ ), there is no possibility of interpretation at multivariate level. While OBLBP "mix" together the spectral and spatial information, GHOST embeds them in different dimensions as probabilistic measures, thus enabling a intuitive analysis. Thanks to its implementation using FVG and KLPD, GHOST is processed in a full-band manner without the need for dimensionality reduction at the spectral level. This preserves data integrity and the underlying spectral-spatial details which is crucial for metrology.

Our next work is to devise a multiscale analysis to consider texture at different scales as well as a multireference formulation to improve spectral discriminability. Alternatives to GMM modeling will also be studied for more efficient similarity assessment between high-dimensional distributions.

\section{CONCLUSION}

We have devised a gradient-based texture feature using fullband processing for hyperspectral image. The formulation is based on the proposed definition of texture as joint spectral and spatial distribution. The resulted feature, GHOST is expressed as a four-dimensional probability density distribution. Thanks to the metrological gradient and spectral difference calculation, the formulation is generic and applicable for images of any spectral band and range. Intuitive and rich in information, GHOST jointly measures texture "magnitude", direction and spectral distribution. We have validated our approach via a HyTexiLa classification scheme. GHOST registers comparable performance with OBLBP, attaining an accuracy of $91 \%-99 \%$ with feature size $0.2 \%$ of OBLBP's.

\section{REFERENCES}

[1] A. H-Heurtier, "Texture feature extraction methods: A survey," IEEE Access, vol. 7, pp. 8975-9000, 2019.

[2] B. Julesz, "Visual pattern discrimination," IRE Trans. on Info. Theory, vol. 8, no. 2, pp. 84-92, 1962.

[3] B. Julesz, E. Gilbert, and J. Victor, "Visual discrimination of textures with identical third-order statistics," Biological cybernetics, vol. 31, pp. 137-40, 011979.
[4] Persi Diaconis and David Freedman, "On the statistics of vision: The julesz conjecture," Journal of Mathematical Psychology, vol. 24, no. 2, pp. 112 - 138, 1981.

[5] J. I. Yellott, "Implications of triple correlation uniqueness for texture statistics and the julesz conjecture," JOSA A, vol. 10, no. 5, pp. 777-793, 1993.

[6] H. Chatoux, N. Richard, F. Lecellier, and C. FMaloigne, "Gradient in spectral and color images: from the di zenzo initial construction to a generic proposition," JOSA A, vol. 36, no. 11, pp. C154-C165, 2019.

[7] L. Jin, H. Liu, X. Xu, and E. Song, "Improved direction estimation for di zenzo's multichannel image gradient operator," Pattern Recognit., vol. 45, no. 12, 2012.

[8] C. Harris and M. Stephens, "A combined corner and edge detector," in Procedings of the Alvey Vision Conference 1988. 1988, Alvey Vision Club.

[9] S. Di Zenzo, "A note on the gradient of a multi-image," Computer Vision, Graphics, and Image Processing, vol. 33 , no. 1 , pp. $116-125,1986$.

[10] G. Sapiro, “Color snakes," Computer Vision and Image Understanding, vol. 68, no. 2, pp. 247 - 253, 1997.

[11] J. Shi and C. Tomasi, "Good features to track," in 1994 Proc. of IEEE CVPR, 1994, pp. 593-600.

[12] N. Richard, D. Helbert, C. Olivier, and M. Tamisier, "Pseudo-Divergence and Bidimensional Histogram of Spectral Differences for Hyperspectral Image Processing," Journal of Imaging Science and Technology, 2016.

[13] R. J. Chu, N. Richard, F. Ghorbel, C. F-Maloigne, and J. Y. Hardeberg, "A metrological framework for hyperspectral texture analysis using relative spectral difference occurrence matrix," in 2019 10th WHISPERS, 2019, pp. 1-5.

[14] S. Kullback and R. A. Leibler, "On information and sufficiency," The Annals of Mathematical Statistics, vol. 22, no. 1, pp. 79-86, 1951.

[15] J. R. Hershey and P. A. Olsen, "Approximating the kullback leibler divergence between gaussian mixture models," in IEEE ICASSP, 2007, vol. 4, pp. IV-317-IV-320.

[16] H. A. Khan, S. Mihoubi, B. Mathon, J. B. Thomas, and J. Y. Hardeberg, "Hytexila: High resolution visible and near infrared hyperspectral texture images," Sensors, vol. 18 , no. $7,2018$. 